\title{
Câncer de mama em mulheres no Maranhão: estudo de sobrevida no Centro de Assistência de Alta Complexidade em Oncologia (CACON) em São Luís - MA (1998-2004)
}

Breast cancer in women of the state of Maranhão, Brazil: a survival study in an Oncology Center at São Luís - MA (1998-2004)

Autora: Dulcelena Ferreira Silva

Orientadoras: Profa. Dra. Maria do Desterro Soares Brandão Nascimento, Profa. Dra.Luciane Maria Oliveira Brito

Dissertação apresentada ao Programa de Pós-Graduação em Ciências da Saúde da Universidade Federal do Maranhão, para obtenção do Título de Mestre, em 28 de abril de 2006.

Objetivos: estudar a sobrevida de mulheres com câncer de mama tratadas no Centro de Assistência de Alta Complexidade em Oncologia/Instituto Aldenora Bello (CACON/IMOAB) em São Luís - MA. Material e Métodos: foi realizado um estudo descritivo com avaliação de 103 prontuários médicos com registro de óbito por câncer de mama no CACON/IMOAB. Foram levantados dados sóciodemográficos, estadiamento clínico, tipo histológico, grau de diferenciação do tumor, tratamento, tempo de sobrevida em função do estadiamento inicial do tumor. Para análise estatística, foi ajustada uma regressão linear múltipla dos dados. Foi considerado um nível de significância de 5\%. Resultados e Conclusões: o câncer de mama foi mais freqüente nas mulheres entre 40 e 49 anos (38,8\%), solteiras (45,6\%), com escolaridade de 4 a 11 anos $(47,6 \%)$, procedentes da capital, São Luís (68,9\%). O estadiamento clínico mais comum foi o tipo III $(51,5 \%)$, avançado. Os tipos histológicos e graus de diferenciação mais comuns, respectivamente, foram o carcinoma ductal infiltrante $(82,5 \%)$ e o moderadamente diferenciado $(60,7 \%)$. A associação terapêutica mais comumente empregada (40,8\%) foi o tratamento cirúrgico, quimio e radioterapia. O estadiamento no momento da admissão foi a única variável explanatória que se revelou significante na determinação da sobrevida $(p=0,004)$ O estadiamento I teve sobrevida mediana de 13 anos, ao passo que os tipos III e IV ficaram entre um e dois anos. Estas informações propõem que sejam instituídas ações em saúde pública objetivando diagnóstico precoce e terapêutica adequada.

PALAVRAS-CHAVE: Câncer de mama; Câncer de mama localmente avançado; Sobrevida; Epidemiologia

\section{Efeito analgésico da estimulação elétrica nervosa transcutânea na dor pélvica de mulheres com endometriose pélvica}

Analgesic effect of transcutaneous electrical nerve stimulation on pelvic pain in women with endometriosis Autora: Edmara Rita Telles

Orientadora: Profa. Dra Vivian Ferreira do Amaral

Dissertação de Mestrado apresentada ao Programa de Pós Graduação em Ciências da Saúde da Pontifícia Universidade Católica do Paraná (CCBS-PUCPR), em 11de agosto de 2006.

Objetivo: determinar a eficácia da analgesia induzida pela Estimulação Elétrica Transcutânea (TENS) nas dores pélvicas de mulheres com endometriose pélvica. Desenho do estudo: realizou-se um estudo prospectivo, randomizado e cego, de 42 mulheres com endometriose pélvica, comprovada por videolaparoscopia e biópsia, alocadas em 2 grupos assim dispostos: o grupo TENS foi composto por 22 mulheres, submetidas ao tratamento da TENS no modo burst, durante 27 sessões de $30 \mathrm{~min}$; o grupo controle, caracterizado por 20 mulheres submetidas ao uso do TENS através do mesmo protocolo, mas sem a passagem de corrente terapêutica (placebo). A dor pélvica foi quantificada pela Escala Visual Numérica (EVN) antes e após cada sessão, pelo questionário de McGill e algometria, antes, durante e depois de trinta dias do término do tratamento. Para a análise estatística aplicou-se o teste Mann-Whitney, e Friedman e, para as variáveis dicotômicas, o teste exato de Fisher.

Resultados: observou-se uma redução significativa da dor depois de cada aplicação da TENS, ao término das 27 sessões e depois de trinta dias do término do tratamento ( $p<0,04, p<0,001$ e $p<0,05$, respectivamente) do grupo TENS em relação ao grupo controle.

Conclusão: o uso da TENS é eficaz no tratamento e no controle imediato da dor pélvica em mulheres com endometriose pélvica.

PALAVRAS-CHAVE: Estimulação elétrica transcutânea; Endometriose pélvica; Dor pélvica; Tratamento conservador 